\title{
Spin dephasing and photoinduced spin diffusion in a high-mobility two-dimensional electron system embedded in a GaAs-(Al,Ga)As quantum well grown in the [110] direction
}

\author{
R. Völkl, ${ }^{1}$ M. Griesbeck, ${ }^{1}$ S. A. Tarasenko, ${ }^{2}$ D. Schuh, ${ }^{1}$ W. Wegscheider, ${ }^{3}$ C. Schüller,${ }^{1}$ and T. Korn ${ }^{1, *}$ \\ ${ }^{1}$ Institut für Experimentelle und Angewandte Physik, Universität Regensburg, D-93040 Regensburg, Germany \\ ${ }^{2}$ A. F. Ioffe Physical-Technical Institute, Russian Academy of Sciences, 194021 St. Petersburg, Russia \\ ${ }^{3}$ Solid State Physics Laboratory, ETH Zurich, 8093 Zurich, Switzerland \\ (Received 16 May 2011; published 24 June 2011)
}

\begin{abstract}
We have studied spin dephasing and spin diffusion in a high-mobility two-dimensional electron system, embedded in a GaAs/AlGaAs quantum well grown in the [110] direction, by a two-beam Hanle experiment. For very low excitation density, we observe spin lifetimes of more than $16 \mathrm{~ns}$, which rapidly decrease as the pump intensity is increased. Two mechanisms contribute to this decrease: The optical excitation produces holes, which lead to a decay of electron spin via the Bir-Aronov-Pikus mechanism and recombination with spin-polarized electrons. By scanning the distance between the pump and probe beams, we observe the diffusion of spin-polarized electrons over more than $20 \mu \mathrm{m}$. For high pump intensity, the spin polarization in a distance of several micrometers from the pump beam is larger than at the pump spot, due to the reduced influence of photogenerated holes.
\end{abstract}

DOI: 10.1103/PhysRevB.83.241306

PACS number(s): 75.40.Gb, 85.75.-d, 73.61.Ey

Semiconductor spintronics is a research field which aims at utilizing the electron-spin degree of freedom, instead of its charge, for information processing..$^{1,2}$ A key ingredient for spintronics devices is a semiconductor structure, which allows for long-range transport of spin-polarized carriers as well as manipulation of the spin polarization via the Rashba spin-orbit interaction. ${ }^{3}$ While several studies on spin injection and spin transport in GaAs have focused on weakly $n$-doped GaAs bulk layers, ${ }^{4-6}$ the mobility in these structures is very low due to the direct doping, and the bulklike character of the layer does not allow for direct spin modulation via the Rashba effect. By contrast, in two-dimensional electron systems (2DES) based on modulation doping of quantum wells (QWs), very high mobilities can be reached, and electrical control of the spin dynamics via the Rashba effect has been demonstrated. ${ }^{7}$ Structures grown along the [110] crystallographic direction are particularly promising for spintronics applications, as the Dresselhaus spin-orbit field points along the growth direction and spin dephasing via the Dyakonov-Perel (DP) mechanism ${ }^{8}$ is strongly suppressed, ${ }^{9-11}$ or modified, if there is also Rashba spin-orbit interaction due to growth-axis asymmetry. ${ }^{12}$

Here, we present spatially resolved studies of the spin dynamics of optically oriented electrons in a high-mobility 2DES embedded in a (110)-grown GaAs/AlGaAs QW structure. Our sample is a 30-nm-wide QW with electron density $n_{e}=2.4 \times 10^{11} \mathrm{~cm}^{-2}$ and mobility $\mu=2.3 \times 10^{6} \mathrm{~cm}^{2} / \mathrm{V} \mathrm{s}$ at $1.5 \mathrm{~K}$, determined by magnetotransport measurements. The modulation doping is placed symmetrically above and below the QW in a complex structure similar to that used by Umansky et al. ${ }^{13}$ A similar growth procedure leads to even higher carrier mobilities in (001)-oriented QWs and allows for the study of ballistic cyclotron motion. ${ }^{14}$ Due to the low growth temperature for the (110) surface, symmetrical remote doping results in a symmetrical band profile of the QW and vanishing Rashba interaction. ${ }^{15,16}$ The sample is mounted in vacuum on the cold finger of a He-flow cryostat, and the electron temperature in the sample during measurements has been determined by analyzing the high-energy tail of the photoluminescence (see, e.g., Ref. 17 for details) to be $\sim 20 \mathrm{~K}$. A cw diode laser is used as a source for the circularly polarized pump beam. It nonresonantly excites spin-polarized electron-hole pairs in the QW with an excess electron energy $\sim 25 \mathrm{meV}$ above the Fermi energy. The $z \|[110]$ component of the spin polarization is detected by near-resonant probing with a tunable Ti-sapphire laser, which is linearly polarized, via the magneto-optic Kerr effect (MOKE). Both beams are superimposed on each other at a beamsplitter and either coupled into a $20 \times$ microscope objective or focused onto the sample using a lens with 50-mm focal length. The reflected probe beam is spectrally filtered using a bandpass to suppress the collinear pump beam and then coupled into an optical bridge, which detects the small rotation of the probe beam polarization axis due to the polar MOKE. A lock-in modulation scheme is used to increase the sensitivity of the detection. The spot sizes of pump and probe beams are 6 and $3 \mu \mathrm{m}$, respectively, if the microscope objective is used. The lens leads to larger spot sizes of $40 \mu \mathrm{m}$ for both beams. The spot size is determined by scanning the beam over a lithographically defined structure. For spatially resolved measurements, the pump beam is scanned with respect to the probe beam by a piezocontrolled mirror. The cryostat is mounted between a pair of Helmholtz coils, and magnetic fields up to $30 \mathrm{mT}$ can be applied in the sample plane.

First, we discuss the electron-spin dephasing at the overlap of pump and probe spots as a function of the pump intensity.

Figure 1(a) shows a series of Hanle-MOKE traces measured for different excitation densities of the pump beam. The traces have been normalized for easier comparison. All traces show the typical Lorentzian line shape of a Hanle curve: At zero magnetic field, the out-of-plane component $S_{z}$ of the total spin $S$ has a maximum. An applied in-plane magnetic field leads to spin precession and a decrease of $S_{z}$. The spin lifetime $\tau_{s}$ can be extracted from the half-width of the Lorentzian, which is described by

$$
S_{z}(B)=\frac{S_{z}(0)}{1+\left(\omega_{L} \tau_{s}\right)^{2}} .
$$



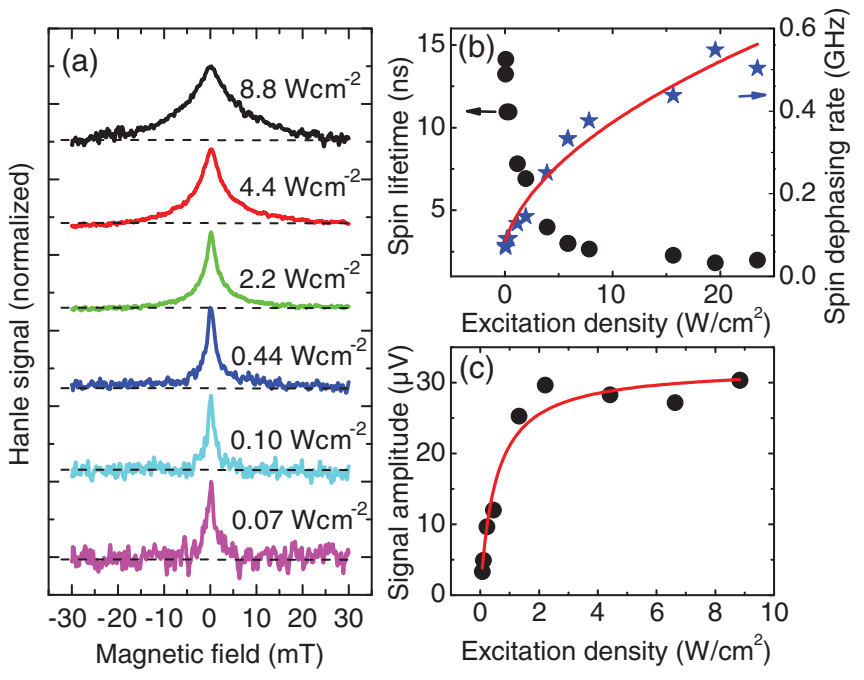

FIG. 1. (Color online) (a) Hanle-MOKE traces measured for different excitation densities. (b) Spin lifetime $\tau_{s}$ (dots) and spin dephasing rate (stars) as a function of excitation density. The solid line is a fit to Eq. (8) with $I_{0}=0.53 \mathrm{~W} / \mathrm{cm}^{2}$. (c) Hanle-MOKE signal amplitude as a function of the excitation density. The solid line is plotted after Eq. (5) with the same $I_{0}$.

Here, $\omega_{L}=g_{e} \mu_{B} B / \hbar$ is the Larmor frequency, which is determined by the electron $g$ factor and the applied magnetic field $B$, and $\mu_{B}=e \hbar / 2 m$ is the Bohr magneton. The in-plane $g$ factor of our sample has been determined by time-resolved Faraday rotation measurements (not shown) to be $\left|g_{e}\right|=0.38$. The series of Hanle-MOKE traces clearly shows how the Lorentzian widens with increasing the excitation density of the pump beam. Figure 1(b) shows the extracted spin lifetime and the corresponding dephasing rate $1 / \tau_{s}$ as a function of the excitation density. For the lowest excitation density used, we find a spin lifetime of $14.1 \mathrm{~ns}$, which is of the same order as values recently observed for (110)-grown QWs by spin noise spectroscopy ${ }^{18,19}$ in small in-plane magnetic fields. As the excitation density is increased, the spin lifetime drops significantly, and the dephasing rate increases accordingly. A similar behavior of the spin lifetime as a function of excitation density is observed in measurements with a large focal spot of $40 \mu \mathrm{m}$ (not shown). The larger total laser power used in these measurements allows us to reach even lower excitation densities, resulting in a maximum observed $\tau_{s}$ of $16.4 \mathrm{~ns}$.

We also analyze the amplitudes of the Hanle curves, which provide information on the spin polarization at zero magnetic field. It is observed that the amplitude, depicted in Fig. 1(c), saturates for excitation densities above $1 \mathrm{~W} \mathrm{~cm}^{-2}$.

In order to interpret both observations, the decrease of $\tau_{s}$ and the saturation of $S_{z}(0)$ with the excitation density, we consider the spin dynamics of optically oriented electrons. For simplicity, we neglect the influence of inhomogeneous pumping and (spin) diffusion on the spin dynamics. This is justified, as our measurements using a large focal diameter spot yield similar values for the spin dephasing rates and show the same functional dependence on intensity as the measurements using the smaller focal diameter.
At zero magnetic field, the $z$ component of the steady-state total electron spin is given by

$$
S_{z}(0)=G_{z} \tau_{z}
$$

where $G_{z}$ is the spin generation rate, proportional to the excitation density $I$, and $\tau_{z}$ is the lifetime of electron spin oriented along the QW normal. The decay rate $1 / \tau_{z}$ is determined by three contributions,

$$
1 / \tau_{z}=1 / \tau_{z}^{\lim }+\gamma_{z}^{\mathrm{BAP}} N_{h}+\gamma^{r} N_{h},
$$

with $1 / \tau_{z}^{\lim }$ being the spin dephasing rate in the limit of zero excitation. In this regime, spin dephasing may occur either due to the Elliott-Yafet mechanism $(E Y)^{20}$ or due to the DP mechanism caused by small random Rashba fields in a modulation-doped structure. ${ }^{21,22}$ The terms $\gamma_{z}^{\mathrm{BAP}} N_{h}$ and $\gamma^{r} N_{h}$ describe the spin decay due to the Bir-Aronov-Pikus (BAP) mechanism, ${ }^{23}$ which is proportional to the steady-state hole density $N_{h}$, and the recombination of optically oriented electrons with holes, respectively. The role of photocarrier recombination is as follows: In the limit of high excitation density, nearly all of the optically oriented electrons would recombine with holes, similar to an undoped $\mathrm{QW}$, and the photocarrier lifetime limits the spin lifetime. It is assumed here that the recombination probability for an electron is also proportional to the steady-state hole density, which is usually valid for a nondegenerate hole gas in the absence of recombination centers, and $N_{h} \ll n_{e}$. We also note that, for simplicity, we neglect the influence of photoexcitation on the EY and DP mechanisms.

It follows from Eqs. (2) and (3) that $S_{z}(0)$ is given by

$$
S_{z}(0)=\frac{G_{z}}{1 / \tau_{z}^{\lim }+\left(\gamma_{z}^{\mathrm{BAP}}+\gamma^{r}\right) N_{h}} .
$$

In the limit of very low excitation density, where the time $\tau_{z}^{\text {lim }}$ determines the spin lifetime, $S_{z}(0)$ depends linearly on the excitation density $I$, as $G_{z} \propto I$. For increased excitation density, $S_{z}$ saturates, as $N_{h} \propto I$. Due to the low spin dephasing rate $1 / \tau_{z}^{\lim }$, this saturation already occurs at rather low values of the excitation density, as observed in Fig. 1(c). The experimental amplitude data is well described by the fit function

$$
S_{z}(0) \propto \frac{I}{1+I / I_{0}},
$$

which has the excitation density dependence of Eq. (4). We note that, from the excitation-density dependence, the relative magnitude of $\gamma_{z}^{\text {BAP }}$ and $\gamma^{r}$ cannot be determined, but we may conclude that $1 / \tau_{z}^{\lim }$ and $\left(\gamma_{z}^{\mathrm{BAP}}+\gamma^{r}\right) N_{h}$ are already comparable at an excitation level of less than $I \sim 1 \mathrm{~W} / \mathrm{cm}^{2}$.

Next, we consider the spin dephasing in an applied inplane magnetic field. The spin lifetime obtained in Hanle measurements is given by

$$
\tau_{s}=\sqrt{\tau_{z} \tau_{\|}},
$$

where $\tau_{\|}$is the in-plane spin dephasing time. The rate $1 / \tau_{\|}$ can be also presented in the form of Eq. (3). However, $1 / \tau_{\|}^{\lim } \gg 1 / \tau_{z}^{\lim }$ in (110)-grown structures, as $1 / \tau_{\|}^{\lim }$ is determined by the conventional DP mechanism in the Dresselhaus field perpendicular to the QW plane, while $\gamma_{\|}^{\mathrm{BAP}}$ and $\gamma_{z}^{\mathrm{BAP}}$ 
are comparable. For symmetrically (110)-grown structures, $\tau_{z}^{\lim } / \tau_{\|}^{\lim } \approx 7$ has been reported previously. ${ }^{18}$ In the excitation density range we study, we may therefore neglect the effects of the BAP mechanism and recombination on the in-plane spin dephasing rate, and use the following expression for the spin lifetime measured in Hanle experiments:

$$
1 / \tau_{s}=\sqrt{1 / \tau_{\|}^{\lim }} \sqrt{\left[1 / \tau_{z}^{\lim }+\left(\gamma_{z}^{\mathrm{BAP}}+\gamma^{r}\right) N_{h}\right]} .
$$

For $N_{h} \propto I$, the spin dephasing rate extracted from the Hanle curves has the following intensity dependence,

$$
1 / \tau_{s}(I)=1 / \tau_{s}(0) \sqrt{1+I / I_{0}},
$$

with the same intensity $I_{0}$ as in Eq. (5). As Fig. 1(b) shows, the measured rates are in good agreement with Eq. (8). Both the intensity dependence of the spin dephasing rate, and the signal amplitude [Fig. 1(c)], are well described by fits using the value $I_{0}=0.53 \mathrm{~W} \mathrm{~cm}^{-2}$.

Finally, we investigate the spin diffusion in our sample. Figure 2(a) shows a series of scanning Hanle-MOKE measurements. The traces were generated in the following way: At each distance between the pump and probe spot, the difference between the Kerr signals for zero field and 30-mT in-plane fields was recorded, and this amplitude, which is proportional to the local spin polarization, is plotted as a function of the spot distance. For low excitation density, we observe a maximum of the spin polarization at the beam overlap. The spin polarization decays monotonously as the distance between the pump and probe is increased. For higher excitation densities, however, a local minimum becomes visible at the beam overlap, which becomes more pronounced as the excitation density is increased. To quantify this observation, we define an amplitude of this dip as the signal difference between the local minimum at the beam overlap and the adjacent absolute maxima at finite pump-probe beam distance. Spin polarization at the beam overlap saturates below $3 \mathrm{~W} / \mathrm{cm}^{2}$, and above this value
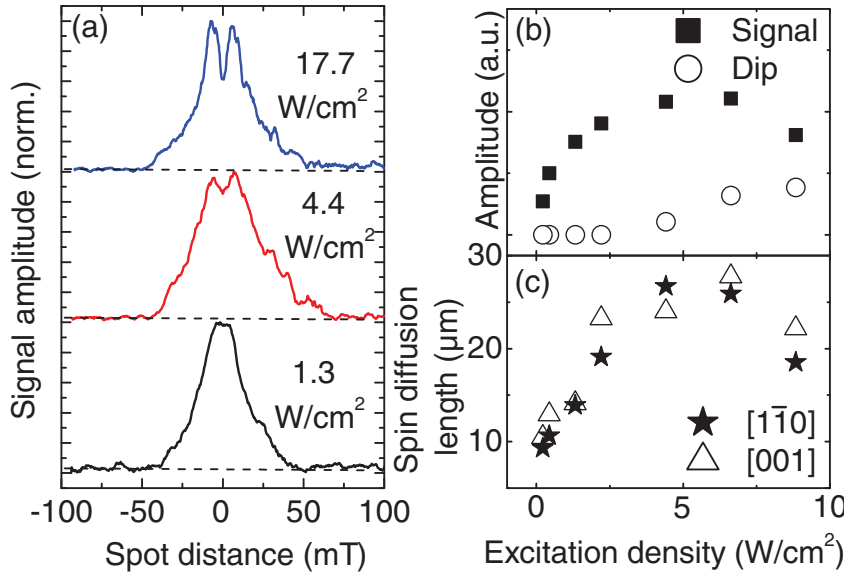

FIG. 2. (Color online) (a) Scanning Hanle-MOKE measurements for different excitation densities. The curves have been normalized for easy comparison. (b) Amplitude of the Hanle-MOKE signal at the beam overlap (squares) and the dip amplitude (circles) as functions of the excitation density. (c) Spin diffusion length as a function of excitation density. The different scan directions are indicated by triangles $([001])$ and stars $([1 \overline{1} 0])$. the dip develops and increases its amplitude, as Fig. 2(b) demonstrates.

This anomalous spin distribution in the QW plane can be explained by different diffusion coefficients of electrons and heavy holes in a GaAs-based QW structure. The lighter spinpolarized electrons rapidly diffuse away from the pump spot due to their low mass, while the heavy holes move more slowly. The electron diffusion is significantly faster in this highmobility 2DES as compared to, e.g., bulk $n$-GaAs, due to the low concentration of momentum scattering sites and the large Fermi wave vector of the electrons. Consequently, in a distance of a few micrometers from the pump spot, which is smaller then the diffusion length of spin-polarized electrons, the hole density is significantly reduced. Therefore, hole-dependent spin dephasing and recombination processes are suppressed, resulting in a larger electron-spin polarization. We note that the width of the observed dip increases only slightly from $\sim 8$ to $11 \mu \mathrm{m}$ as a function of the excitation density, indicating the slow diffusion of the optically created holes. The spin-diffusion length $l_{s}$ (determined by fitting an exponential decay of the spin polarization with distance far from the beam overlap, so that the signal dip may be neglected) increases first with the excitation density, as Fig. 2(c) shows, reaching a maximum of $\sim 27 \mu \mathrm{m}$, then decreases again for higher excitation densities. The two in-plane crystallographic directions [001] [110] show a comparable behavior. Several effects are likely to contribute to the initial increase. First, the inhomogeneous distribution of electrons and holes in the QW plane produces local electric fields. Far away from the pump spot, the field is directed toward the spot and induces a divergent electron flow enlarging the area of spin polarization. Second, with the local increase of the electron density, high- $k$ states are occupied by the optically oriented electrons. Additionally, the rise of spin polarization suppresses electron-electron collisions between particles of opposite spins, which slow down the spin diffusion, ${ }^{24,25}$ thereby increasing $l_{s}$. For very high excitation densities, the spin-diffusion length is decreased due to local heating of the sample and electron-electron scattering. To study the spatial dependence
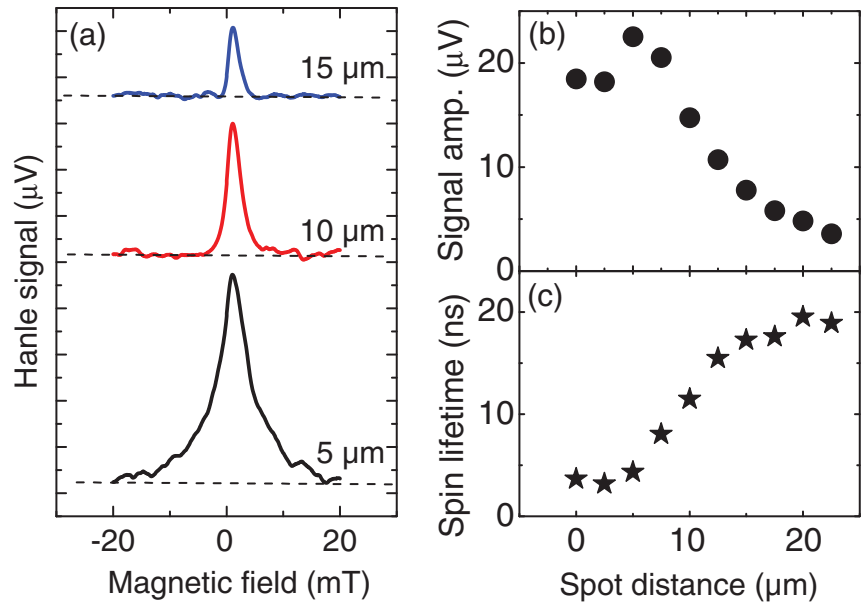

FIG. 3. (Color online) (a) Hanle-MOKE traces for different distances between pump and probe beams. (b) Signal amplitude of the Hanle-MOKE traces as a function of pump-probe distance. (c) Spin lifetime extracted from the Hanle-MOKE traces as a function of pump-probe distance. 
of the spin lifetime in more detail, we perform Hanle-MOKE measurements for a high excitation density $\left(5 \mathrm{~W} \mathrm{~cm}^{-2}\right)$ as a function of the pump and probe beam distance. Figure 3(a) shows a series of Hanle-MOKE traces for different distances between pump and probe. We clearly observe that the Hanle curves become more narrow as the distance increases. This corresponds to an increase of the effective spin lifetime from below $4 \mathrm{~ns}$ at the beam overlap to a saturation value of $\sim 18 \mathrm{~ns}$, which is reached at a distance of $15 \mu \mathrm{m}$ between the pump and probe [Fig. 3(c)]. A spatially inhomogeneous spin lifetime due to local optical pumping was previously observed in bulk $n$-GaAs. ${ }^{6}$ The amplitude of the Hanle signal extracted from these traces, shown in Fig. 3(b), mimics the scanning HanleMOKE measurements for high excitation density [Fig. 2(a)], in that the signal amplitude has a dip at the beam overlap and then first increases at a distance of $5 \mu \mathrm{m}$, then decreases again.
In conclusion, we have investigated the spin dephasing and spin diffusion in a high-mobility (110)-grown twodimensional electron system by spatially resolved HanleMOKE measurements. We observe long spin dephasing times at low excitation density, which are strongly reduced for higher excitation. The spatially resolved measurements show a very long spin-diffusion length up to $27 \mu \mathrm{m}$. For high excitation density, the spin polarization is larger at some distance from the pump laser spot than at the overlap of pump and probe beams due to the reduced spin dephasing in the absence of optically created holes.

The authors would like to thank E. L. Ivchenko, M. M. Glazov, J.-H. Quast, T. Kiessling, W. Ossau, and E.Ya. Sherman for fruitful discussions. Financial support by the DFG via SPP 1285 and SFB 689, RFBR, and "Dynasty" Foundation is gratefully acknowledged. *tobias.korn@physik.uni-regensburg.de

${ }^{1}$ I. Zutic, J. Fabian, and S. Sarma, Rev. Mod. Phys. 76, 323 (2004).

${ }^{2}$ M. W. Wu., J. H. Jiang, and M. Q. Weng, Phys. Rep. 493, 61 (2010).

${ }^{3}$ Y. A. Bychkov and E. I. Rashba, J. Phys. C 17, 6039 (1984).

${ }^{4}$ J. Kikkawa and D. Awschalom, Nature (London) 397, 139 (1999).

${ }^{5}$ M. Furis, D. L. Smith, S. Kos, E. S. Garlid, K. S. M. Reddy, C. J. Palmstrøm, P. A. Crowell, and S. A. Crooker, New J. Phys. 9, 347 (2007).

${ }^{6}$ J.-H. Quast, G. V. Astakhov, W. Ossau, L. W. Molenkamp, J. Heinrich, S. Höfling, and A. Forchel, Phys. Rev. B 79, 245207 (2009).

${ }^{7}$ O. Z. Karimov, G. H. John, R. T. Harley, W. H. Lau, M. E. Flatte, M. Henini, and R. Airey, Phys. Rev. Lett. 91, 246601 (2003).

${ }^{8}$ M. Dyakonov and V. Perel, Sov. Phys. JETP 33, 1053 (1971).

${ }^{9}$ M. Dyakonov and V. Kachorovskii, Sov. Phys. Semicond. 20, 110 (1986).

${ }^{10}$ Y. Ohno, R. Terauchi, T. Adachi, F. Matsukura, and H. Ohno, Phys. Rev. Lett. 83, 4196 (1999).

${ }^{11}$ S. Döhrmann, D. Hägele, J. Rudolph, M. Bichler, D. Schuh, and M. Oestreich, Phys. Rev. Lett. 93, 147405 (2004).

${ }^{12}$ S. A. Tarasenko, Phys. Rev. B 80, 165317 (2009).

${ }^{13}$ V. Umansky, M. Heiblum, Y. Levinson, J. Smet, J. Nübler, and M. Dolev, J. Cryst. Growth 311, 1658 (2009).
${ }^{14}$ M. Griesbeck, M. M. Glazov, T. Korn, E. Y. Sherman, D. Waller, C. Reichl, D. Schuh, W. Wegscheider, and C. Schüller, Phys. Rev. B 80, 241314 (2009).

${ }^{15}$ V. V. Bel'kov, P. Olbrich, S. A. Tarasenko, D. Schuh, W. Wegscheider, T. Korn, C. Schüller, D. Weiss, W. Prettl, and S. D. Ganichev, Phys. Rev. Lett. 100, 176806 (2008).

${ }^{16}$ P. Olbrich, J. Allerdings, V. V. Bel'kov, S. A. Tarasenko, D. Schuh, W. Wegscheider, T. Korn, C. Schüller, D. Weiss, and S. D. Ganichev, Phys. Rev. B 79, 245329 (2009).

${ }^{17}$ T. Korn, Phys. Rep. 494, 415 (2010).

${ }^{18}$ G. M. Müller, M. Römer, D. Schuh, W. Wegscheider, J. Hübner, and M. Oestreich, Phys. Rev. Lett. 101, 206601 (2008).

${ }^{19}$ G. M. Müller, M. Oestreich, M. Römer, and J. Hübner, Physica E 43, 569 (2010).

${ }^{20}$ Y. Yafet, Solid State Physics, Vol. 14 (Academic, New York, 1963).

${ }^{21}$ M. M. Glazov and E. Y. Sherman, Phys. Rev. B 71, 241312 (2005).

${ }^{22}$ Y. Zhou and M. W. Wu, Europhys. Lett. 89, 57001 (2010).

${ }^{23}$ G. L. Bir, A. G. Aronov, and G. E. Pikus, Sov. Phys. JETP 42, 705 (1975).

${ }^{24}$ I. D'Amico and G. Vignale, Phys. Rev. B 62, 4853 (2000).

${ }^{25}$ C. P. Weber, N. Gedik, J. E. Moore, J. Orenstein, J. Stephens, and D. D. Awschalom, Nature (London) 437, 1330 (2005). 